\title{
The Role of Long-Chained Marine N-3 Polyunsaturated Fatty Acids in Cardiovascular Disease
}

\author{
Hildegunn Aarsetoey, ${ }^{1}$ Heidi Grundt, ${ }^{1,2}$ Ottar Nygaard, ${ }^{2,3}$ and Dennis W. T. Nilsen ${ }^{2,4}$ \\ ${ }^{1}$ Department of Medicine, Stavanger University Hospital, 4011 Stavanger, Norway \\ ${ }^{2}$ Institute of Medicine, University of Bergen, 5020 Bergen, Norway \\ ${ }^{3}$ Department of Heart Disease, Haukeland University Hospital, 5021 Bergen, Norway \\ ${ }^{4}$ Department of Cardiology, Stavanger University Hospital, 4011 Stavanger, Norway \\ Correspondence should be addressed to Heidi Grundt, heidi@madlalia.no
}

Received 10 June 2012; Revised 11 October 2012; Accepted 25 October 2012

Academic Editor: Frederic Kontny

Copyright ( $) 2012$ Hildegunn Aarsetoey et al. This is an open access article distributed under the Creative Commons Attribution License, which permits unrestricted use, distribution, and reproduction in any medium, provided the original work is properly cited.

\begin{abstract}
This paper reviews the current evidence regarding long-chained marine omega-3 polyunsaturated fatty acids (PUFAs) and cardiovascular disease (CVD), their possible mechanisms of action, and results of clinical trials. Also, primary and secondary prevention trials as studies on antiarrhythmic effects and meta-analyses are summarized. However, the individual bioavailability of n-3 PUFAs along with the highly different study designs and estimations of FAs intake or supplementation dosages in patient populations with different background intake of n-3 PUFAs might be some of the reasons for the inconsistent findings of the studies evaluating the impact of n-3 PUFAs on CVD. The question of an optimum dose of $n-3$ PUFAs or whether there exists a dose-response relation for n-3 PUFA supplementation is widely discussed. Moreover, the difficulties in interpreting meta-analyses are clearly demonstrated by two recently published meta-analyses (Rizos et al. and Delgado Lista et al.), evaluating the efficacy of n-3 PUFAs on CVD, including 12 common studies, but drawing opposite conclusions. We definitely need more large-scale, randomized clinical trials of long duration, also reporting harmful effects of n-3 PUFAs.
\end{abstract}

\section{Introduction}

Unsaturated fatty acids (FAs), especially polyunsaturated fatty acids (PUFAs), have since the 1970s been given a lot of attention due to possible health promoting effects. During the first decade of research, pilot studies on Greenland Eskimos demonstrated that a diet rich in long-chained marine PUFAs might reduce the incidence of ischemic heart disease [1]. During the following decades, research has to a large extent focused on the prevention and management of cardiovascular disease (CVD).

During the same period of time, there has been a large change in our understanding of the atherosclerotic process. From being viewed as inanimate tubes, arteries are now thought of as dynamic tissues, where intimal inflammation plays a crucial role in the pathophysiologic process of atherosclerotic development. A thin fibrous cap is the only structure separating the blood compartment with its coagulation factors from the prothrombotic material in the lipid core. Enhanced inflammation might result in plaque instability. Moreover, the endothelium plays a key role in vascular homeostasis, and endothelial dysfunction seems to be of major importance in the development of a vulnerable plaque. The acute coronary syndrome (ACS) usually results from the erosion or rupture of such a vulnerable atherosclerotic plaque with subsequent coronary artery occlusion as coagulation factors come into contact with tissue factor, the major initiator of the extrinsic coagulation cascade [2].

The complex vascular biology preceding the ACS provides several possible therapeutic targets for PUFAs: reducing atherosclerotic development, stabilizing vulnerable plaques, and limiting the consequences of their disruption. Even though a lot of research has been done trying to elucidate the role of PUFAs in CVD prevention and management, several issues are still under discussion. Clinical studies have provided conflicting results, and the optimal intake of PUFAs 
is not firmly established. Concerns have been raised about environmental contaminants accumulating in fish, especially methylmercury, polychlorinated biphenyls, and dioxins, giving rise to possible deleterious effects [3] and counteracting the beneficial cardioprotective effects of marine n-3 PUFAs [4]. Especially mercury has been given a lot of attention due to reports of a probable proatherosclerotic effect [5]. N3 PUFAs might also have a potential to increase oxidative stress, resulting in lipid peroxides [6]. These issues might be related to a dose optimum of n-3 PUFAs, as high doses might exceed an optimum threshold level leading to lack of beneficial effects due to lipid peroxidation or accumulation of other toxic substances.

This paper reviews the current evidence regarding longchained marine omega-3 PUFAs and CVD, their possible mechanisms of action, and results of clinical trials.

1.1. PUFAs-Chemistry and Origin. FAs are single-lipid components comprised of a straight hydrocarbon chain terminating with a carboxylic acid group $(-\mathrm{COOH})$ at the polar hydrophilic end and a nonpolar hydrophobic methyl group $\left(-\mathrm{CH}_{3}\right)$ at the other end. The various FAs are named according to their number of carbon atoms and their number and position of carbon-carbon double bonds. FAs with at least two double bonds are designated as polyunsaturated. For PUFAs, the position of the first double bond from the methyl- (n/omega-) end of the molecule has given rise to the terminology n-3 (omega-3) FAs, n-6 (omega-6) FAs, and n-9 (omega-9) FAs [7]. According to the accepted terminology, the number of carbon atoms in a PUFA molecule is designated by the first figure, while the number of double bonds is given by the second figure.

$\mathrm{N}-3$ and n-6 PUFAs are not synthesized by the human body, as they are essential FAs that need to be ingested [8]. While n-6 PUFAs and $\alpha$-linolenic acid $(18: 3 n-3)$ are found in vegetable foods, n-3 PUFAs with more than 20 carbon atoms are made by phytoplankton and mainly ingested from fatty fish and marine animals. The two most important marine FAs with respect to human health are thought to be eicosapentaenoic acid (EPA; $20: 5 n-3)$ and docosahexaenoic acid (DHA; 22:6 n-3). Even though these two FAs to a small extent also can be derived from $\alpha$-linolenic acid by desaturation and elongation in the liver [8], the main body content of EPA and DHA is dependent on the amount ingested. For the present paper we will focus on these longchained marine n-3 PUFAs.

\section{Mechanisms of Action of Long-Chained Marine N-3 PUFAs}

The observed health effects of n-3 PUFAs are mainly thought to be mediated by two mechanisms: a change in the properties of the cell membranes and the regulation of gene transcription. As FAs are incorporated into cell membrane phospholipids, the FA composition of this lipid bilayer is reflected by the composition of FAs ingested. Increasing the amounts of n-3 PUFAs in the cell membrane alters its biochemical and physical properties with a subsequent change of membrane fluidity, permeability, and electrophysiological characteristics [9]. Changing membrane properties might further affect the ability of membrane receptors to interact with their ligands or intracellular signalling molecules as well as modulate the effect of membrane bound enzymes [10]. There is also evidence for n-3 PUFAs themselves serving as ligands for nuclear receptors affecting gene expression, nuclear factor $\kappa \mathrm{B}(\mathrm{NF}-\kappa \mathrm{B})$, and peroxisome proliferatoractivated receptor- $\gamma$ (PPAR- $\gamma$ ) being two of the potential targets [11-13].

Furthermore, 20-carbon PUFAs (arachidonic acid (AA) and EPA) released from cell membrane phospholipids by phospholipase A2 are substrates for the synthesis of eicosanoids, a family of biochemical mediators consisting of prostaglandins (PGs), thromboxanes (TXs), leukotrienes (LTs), and hydroxy fatty acids. Eicosanoids possess important vasoactive regulatory properties, such as regulation of platelet aggregability, endothelial cell motility, cell growth, and chemotaxis $[8,14,15]$. With the increasing incorporation of n-3 PUFAs into cell membranes, the production of eicosanoids is shifted from the 4 series of LTs and the 2 series of PGs and TXs generated from AA to the 5 series of LTs and the 3 series of PGs and TXs derived from EPA, the latter being eicosanoid products leading to a more vasodilatory state, reduced inflammatory responses in the injured vessel wall, and less potent platelet aggregation $[8,14,15]$.

A combination of these mechanisms, summarized in Table 1, seems to be responsible for the majority of the proposed antiatherothrombogenic and antiarrhythmic effects of n-3 PUFAs $[8,14,16,17]$ further outlined below.

\subsection{Marine N-3 PUFAs and Anti-Atherothrombogenic Effects.} In the early 1990s, the extent of atherosclerotic lesions in the coronary arteries and the aortas from Alaskan natives were demonstrated to be significantly lower as compared to non-natives in all age-groups [18]. This was one of the first indications of a possible antiatherosclerotic effect of n-3 PUFAs. In accordance with this finding, a dietary related incorporation of EPA and DHA into advanced human atherosclerotic plaques has been demonstrated $[19,20]$ along with the development of a more stable plaque morphology, less susceptible to rupture, after n-3 PUFA supplementation [20]. The results from intervention studies on coronary atherosclerosis progression and regression in humans have, however, been highly diverging [21-23].

The potential effect on atherosclerosis was initially thought to be related to the modulation of proatherosclerotic risk factors. As compared to Danes, Greenland Inuits had lower serum concentrations of total- and low-density lipoprotein cholesterol, triglycerides, and very low-density lipoprotein cholesterol but higher levels of high density lipoprotein cholesterol $[1,24]$. Since then, several studies have confirmed that the increasing amounts of n-3 PUFAs are associated with a more favourable profile of lipoproteins [8]. The clear-cut triglyceride-lowering effect of marine n-3 PUFA especially is undisputed [8]. Several mechanisms may contribute to the triglyceride-lowering effect by marine n3 PUFAs, such as reduced triglyceride synthesis and diminished chylomicron secretion from intestinal cells. Reduced 
TABLE 1: Mechanisms and biochemical effects of marine PUFA.

Anti-inflammatory effects

(i) Competition with AA for Cox/lipooxygenase sites

(ii) Increase of anti-inflammatory eicosanoids

(iii) Reduction of TNF $\alpha$, IL-1, IL-6

(iv) Reduction of nuclear factor $\kappa \mathrm{B}(\mathrm{NF}-\kappa \mathrm{B})$ activation

Vascular effects

(i) Increased vagal tone

(ii) Improved endothelial function

(iii) Increase of $\mathrm{NO}$

(iv) Reduction of Hcy, VCAM-1, ELAM-1, and ICAM-1

(v) Reduction of ET-1

Antithrombotic effects

(i) Reduced platelet aggregation via reduction in TXA2

(ii) Increased bleeding time (high doses)

Triglyceride-lowering effect

Antiarrhythmic effects

(i) Increased membrane stabilization, reduced automaticity, and increased refractory period

(ii) Increased EPA : AA ratio in plasma membrane of cardiac myocytes

(iii) Reduced production of proarrhythmic eicosanoids

(iv) Reduced agonist affinity of beta-receptors $\rightarrow$ reduced heart rate (HR), increased HR variability

(v) Inhibition of the L-type calcium current

(vi) Inhibition of fast voltage-dependent $\mathrm{Na}^{+}$channels

triglyceride formation may partly be due to a reduced pool of fatty acids substrate mediated through suppressed hepatic fatty acid synthesis related to the effect by marine n-3 PUFAs on hepatic gene expression downregulating de novo lipogenesis, increased fatty acid beta-oxidation, and reduced delivery of nonesterified fatty acids to the liver. Besides, marine n-3 PUFAs are poor substrates for enzymes responsible for triglyceride synthesis resulting in reduced and impaired hepatic enzyme activity for triglyceride synthesis and increased hepatic synthesis of phospholipids rather than triglycerides, hereby limiting the secretion from the liver of triglyceride-rich very low-density lipoprotein (VLDL) [8].

Furthermore, ingestion of n-3 PUFAs has been demonstrated to have a blood pressure reducing effect [25], as well as improving glucose metabolism/insulin resistance [26, 27]. The effect of n-3 PUFAs on insulin as a mediator of the metabolic syndrome needs to be clarified, but there is some support for a relationship between the content of n-3 PUFAs in cell membranes and the action of insulin [8].

Lately, the role of inflammation and endothelial dysfunction in the pathophysiologic process of atherosclerosis generation and disruption of the vulnerable atherosclerotic plaque has been highlighted [28], opening a new area for potential antiatherosclerotic effects of n-3 PUFAs. The first demonstrated anti-inflammatory effects were related to a shift in the production of eicosanoids by partial replacement of AA by EPA in inflammatory cell membranes, as previously described $[8,14,15]$. During recent years, other antiinflammatory effects of $n-3$ PUFAs, independent of the altered eicosanoid production, have been demonstrated. This comprises a reduced production of the proinflammatory cytokines interleukin-1 (IL-1), IL-6, and tumour necrosis factor- $\alpha$ (TNF- $\alpha$ ) from mononuclear cells as well as decreased expression of E-selectin, intercellular adhesion molecule-1 (ICAM-1), and vascular cell adhesion molecule1 (VCAM-1) on endothelial cells [8], the latter being the molecules essential for the attachment of leucocytes to the endothelium prior to their entrance into the intima. Cellular adhesion molecules are markers of the functional state of the endothelium, and their downregulation by n-3 PUFAs have mainly been demonstrated in studies ex vivo. Moreover, beneficial effects on the vasoregulatory secretagogues of the endothelium, such as nitric oxide and prostacycline, have been obtained by $n-3$ PUFAs [8].

The first studies from Bang and Dyerberg on Greenland Eskimos also revealed an antithrombotic effect of high doses of n-3 PUFAs associated with increased bleeding time [29, 30]. However, ingestion of less than 4 grams daily has not been associated with increased risk of bleeding $[8,15,31]$. $\mathrm{TXA}_{2}$ is a potent prothrombotic agent, and it is therefore likely that the observed antiplatelet effect of n-3 FAs, at least to some extent, is mediated through a shift in the eicosanoid production. Homocysteine (Hcy) may also exert unfavourable effects on the antithrombotic properties of the endothelium, and in a randomized trial previously presented by our group we observed a reduction of Hcy after treatment with a high dose of n-3 PUFAs as compared to corn oil for 1 year following a myocardial infarction (MI) [32]. The effect of these PUFAs on other haemostatic procoagulant and fibrinolytic factors is, however, divergent $[16,33,34]$, and the final impact of n-3 PUFA supplementation on the complex processes of vascular injury, thrombosis, and repair still remains an unsettled issue.

2.2. Marine N-3 PUFAs and Antiarrhythmic Effects. The reduction observed in cardiac death with increased intake of $n-3$ PUFAs has largely been attributed to a potential antiarrhythmic effect. Studies on cell cultures have revealed that this might be related to a membrane stabilizing effect in cardiac myocytes. Supplementation with n-3 PUFAs has been found to enrich myocyte membranes with EPA and DHA [35-37]. This induces a conformational change of the cell membrane with effects on ion channels and membranebound proteins resulting in a slight hyperpolarization of the cell membrane, increasing the depolarizing stimuli necessary to induce an action potential with subsequently reduced automaticity. Furthermore, n-3 PUFAs affect the transition of the voltage-gated sodium channel with a shift toward more negative membrane voltages, promoting recovery from the inactive state and thereby increasing the refractory period. Both effects make the myocardium less excitable, especially in ischemic tissue. In these cells, the negative potential required to reactivate the $\mathrm{Na}$ channels might not be physiologically obtainable due to the partial depolarization induced by the 
dysfunctional state of the Na/K-ATPases. Thus, n-3 PUFAs might be important for opposing the effect of functional reentry substrates [9]. They are also capable of inhibiting the voltage-dependent inward calcium current during phase 2 of an action potential. In cooperation with possible effects on the $\mathrm{Na} / \mathrm{Ca}$ exchanger and receptors in the sarcoplasmatic reticulum, this might contribute to less intracellular $\mathrm{Ca}$ fluctuations and reduced occurrence of after depolarizations [9].

In addition to these direct effects on the generation and duration of the action potential, other less direct mechanisms of actions have been proposed. There is also evidence for antiarrhythmic effects mediated through a reduced production of proarrhythmic eicosanoids, reduced levels of circulating catecholamines [38], and a reduced agonist affinity of beta-receptors [17]. The latter observation might be one of the mechanisms responsible for an improvement in the cardiac sympathetic-vagal balance, revealed clinically as a reduction in the mean heart rate (HR) [39] as well as an increase in HR variability [40]. Both of these parameters have been demonstrated to be related to the risk of malignant arrhythmias and sudden cardiac death (SCD), with increasing $\mathrm{HR}$ and decreasing heart rate variability being associated with adverse outcomes [41].

Through these mechanisms, n-3 PUFAs seem to be able to interfere with all the proarrhythmogenic mechanisms responsible for the generation of ventricular arrhythmias.

In agreement with these proposed mechanisms, animal experiments have demonstrated a beneficial effect of EPA and DHA on the development of ischemia-induced ventricular arrhythmias [42], whereas results from human studies are more divergent. For ventricular arrhythmias generated by other mechanisms, such as myocardial scaring and heart failure, the same protective effect of n-3 PUFAs might not be present, as suggested by the highly discrepant results of n-3 PUFA supplementation in patients with an implantable cardioverter defibrillator (ICD) [43-47].

\section{The Omega-3 Index}

The omega- 3 index is defined as the content of EPA and DHA in the cell membrane of red blood cells (RBCs), expressed as a weight percentage of total FAs. The omega-3 index correlates highly with the EPA + DHA content in serum, plasma, and whole blood $[48,49]$, but as opposed to these findings, RBC $\mathrm{EPA}+\mathrm{DHA}$ is better correlated to long-term FA intake as evaluated by a food frequency questionnaire (FFQ) and is a more suitable biomarker for the nutritional status of an individual [49]. The half-life of EPA + DHA in RBCs is 4-6 times longer than in serum [50], with concentrations returning to baseline 16 weeks after supplementation [51].

In addition, the omega- 3 index has been found to be highly correlated with cardiac EPA + DHA levels and responds to supplementation in a way very similar to that of myocardial tissue $[35,36]$. With RBCs readily available and easy to analyze, this gives us the opportunity to apply the omega-3 index as a surrogate for cardiac omega-3 status in clinical practice.

\section{N-3 PUFAs and Relation to Cardiovascular Disease}

The individual bioavailability of $n-3$ PUFAs along with highly different designs, estimations of FA intake, or supplementation dosages might be some of the reasons for the inconsistent findings of studies evaluating the impact of n-3 PUFAs on CVD. A summary of the existing knowledge will be given below.

4.1. Primary and Secondary Prevention Trials. The first evidence of a possible health effect in humans with a diet rich in n-3 PUFAs came from the pioneer studies on Greenland Eskimos in the 1970s [1], reporting lower coronary mortality in this population than in Danish control subjects. Since then, a huge amount of the literature has been published, further investigating the effects of these marinederived PUFAs on CVD. Two of the most cited studies are the Diet and Reinfarction Trial (DART) investigating the effect on recurrent cardiac events of dietary advice after a recent $\mathrm{MI}$ [52] and the GISSI-Prevenzione trial in which marine-derived n-3 PUFA supplementation was given for the prevention of death, nonfatal MI, and stroke in patients who had survived an MI during the preceding 3 months [53]. In the DART study, 2 years of followup revealed a $29 \%$ reduction in total mortality $(P<0.05)$ among patients advised to eat fatty fish, mainly due to a reduction in deaths from coronary artery disease (CAD). Comparable to this finding, supplementation with $1 \mathrm{~g}$ /day of n-3 PUFAs $(\approx 850 \mathrm{mg} /$ day of EPA and DHA ethyl esters in a ratio of $1: 2$ ) for 3.5 years in the open label GISSI-Prevenzione study reduced the composite endpoint of death, nonfatal MI, and stroke by $15 \%(P=0.023)$. In subgroup analyses, the reduction in relative risk was even greater for cardiovascular death and SCD which was reduced by $30 \%(P=0.024)$ and $45 \%(P=0.01)$, respectively.

It has been argued that the GISSI-Prevenzione trial was not designed to evaluate SCD, and that it had insufficient statistical power. The results of these subgroup analyses should, therefore, be interpreted with caution. Furthermore, there has clearly been an evolvement in the general treatment of CAD from the time of performance of the GISSI trial until today, and it has been argued that supplementation with similar doses of n-3 PUFAs in addition to current guideline-adjusted therapy might not have the same benefit. The participants of the GISSI trial were included in the early 1990s, at a time when medical prescriptions and the use of early coronary revascularization were quite different from today's management. To investigate this question, Rauch et al. conducted the Omega trial, a randomized, placebocontrolled, and double-blind trial evaluating the effects of 1year treatment with $1 \mathrm{~g}$ /day of n-3 PUFAs (380 mg DHA + $460 \mathrm{mg}$ EPA) following an MI [54]. Treatment was initiated 3-14 days after MI in 3851 patients. As compared to the GISSI trial in which $5 \%$ of the patients received coronary revascularization at baseline, $93.8 \%$ of the participants of the Omega trial underwent acute percutaneous coronary intervention (PCI). The Omega trial revealed no effect on 
the rate of $\operatorname{SCD}(P=0.84)$, total death $(P=0.18)$, nonfatal reinfarction, stroke $(P=0.10)$, or revascularization procedures in survivors $(P=0.34)$ after 1 year. The Omega trial might, however, have been underpowered in an era of more aggressive risk factor management.

No effect of n-3 PUFA supplementation could be demonstrated on the risk of major cardiovascular events (fatal and nonfatal CVD and cardiac intervention with PCI or coronary artery bypass grafting (CABG) ) in the Alpha Omega Trial [55] in which 4837 patients receiving state-of-the-art medical therapy after MI were randomized to one of four margarines for 40 months; a margarine supplemented with a combination of EPA and DHA, a margarine supplemented with alpha-linolenic acid (ALA), a margarine supplemented with EPA/DHA and ALA, or a placebo margarine. The margarine compounds resulted in an uptake of $226 \mathrm{mg}$ of EPA combined with $150 \mathrm{mg}$ of DHA, $1.9 \mathrm{~g}$ of ALA, or both, respectively. As compared to placebo, neither EPA/DHA nor ALA reduced the occurrence of the primary endpoint (HR with EPA/DHA 1.01 (95\% CI 0.87-1.17; $P=0.93)$; HR with ALA $0.91(95 \%$ CI $0.78-1.05, P=0.20))$. However, the dose of marine n-3 PUFA used in this study was less than half of the dose ingested in the GISSI trial [53].

As opposed to these studies, the GISSI-HF trial provided evidence for a small, but beneficial, advantage of n-3 PUFA supplementation on risk of total mortality (HR 0.91, 95.5\% CI $0.833-0.998, P=0.041$ ) and combined risk of total mortality or admission to hospital for cardiovascular reasons (HR 0.92, 99\% CI 0.849-0.999, $P=0.009$ ) in patients with heart failure of any cause and irrespective of left ventricular ejection fraction. These patients were given standard care according to guidelines in the early 2000s [56]. In absolute terms, 56 patients needed to be treated for a median duration of 3.9 years to avoid one death or 44 to avoid one combined event of death or admission to hospital for cardiovascular reasons. In this randomized, double-blind, and placebocontrolled trial, patients were given $1 \mathrm{~g} \mathrm{n}-3$ PUFAs daily $(n=3494)$ or placebo $(n=3481)$ and followed for a median of 3.9 years. Most interestingly, the same study has demonstrated that n-3 PUFAs can provide a small but significant improvement of left ventricular function in patients with symptomatic heart failure [57]. Left ventricular ejection fraction increased with n-3 PUFAs by $8.1 \%, 11.1 \%$, and $11.5 \%$ after 1,2 , and 3 years, respectively. In the placebo group, the corresponding changes from baseline were $6.3 \%$, $8.2 \%$, and $9.9 \%(P=0.005)$. This finding has recently been verified in two smaller groups of patients with nonischemic dilated cardiomyopathy receiving $1-4 \mathrm{~g} /$ day of $n-3$ PUFAs versus placebo for 3-12 months [58, 59]. In one of these studies, administrating supplements for only 3 months, a dose-dependent increase in left ventricular function was observed [58].

As opposed to the DART and GISSI trials, two recent studies from Japan observed the reduction in risk of CAD to be primarily related to nonfatal coronary events. The Japan Public Health Centre-based (JPHC) study included a total of 41578 Japanese men and women aged 40-59 years initially free of CVD [60]. After 10 years of followup, there was a $57 \%$ reduction in risk of nonfatal cardiac events for the highest as compared to the lowest quintile of fish intake $[\mathrm{HR}=0.43(0.23-0.81)]$, while no favourable effect was observed on fatal events or SCD. In the Japan EPA Lipid Intervention Study (JELIS), supplementation with $1800 \mathrm{mg}$ EPA in combination with a statin was given to 18645 hypercholesterolemic subjects (total cholesterol $\geq$ $6.5 \mathrm{mmol} / \mathrm{L}$ ) for a median time of 4.6 years [61]. Twenty percent of these individuals presented with established CAD. As compared to statin treatment alone, there was a $19 \%$ relative reduction in the primary outcome measure including SCD, fatal, and nonfatal MI and other nonfatal events such as unstable angina pectoris, angioplasty, stenting, and coronary artery bypass grafting $(P=0.011)$. Subgroup analyses demonstrated, however, beneficial effects mainly in the setting of secondary prevention with a significant reduction in risk of unstable angina of $28 \%(P=0.019)$.

The intake of n-3 PUFAs in these Japanese studies was quite high, with participants of the upper quintile of fish intake in the JPHC study having fish servings at least 8 times per week and the population as a whole having a mean intake of $900 \mathrm{mg} \mathrm{n}-3$ PUFAs/day. In a review of prospective cohort studies and randomized controlled trials, Mozaffarian and Rimm [62] demonstrated evidence for a maximal risk reduction of death from $\mathrm{CAD}$ with servings amounting to about $250 \mathrm{mg}$ n-3 PUFAs/day. We recently observed a similar though nonsignificant threshold effect of dietary n-3 PUFAs on risk of coronary events among patients undergoing coronary angiography for suspected coronary artery disease [63]. Thus, for populations already consuming $250 \mathrm{mg}$ /day of EPA + DHA, no further risk reduction for cardiac death seems to be achieved. This threshold-related effect may explain the lack of mortality benefit observed in the JPHC and JELIS studies. Their background fish intake was associated with very low coronary heart disease death rates $(87 \%$ lower than in comparable Western populations), and additional n-3 PUFA intake yielded little further reduction in the death rate, as most of the population was already above the threshold for maximum mortality benefit. In the JPHC study, only subjects with a mean daily intake of $2.4 \mathrm{~g}$ n-3 PUFAs had any reduced risk of nonfatal events, indicating that even greater dosages might be needed to reduce the risk of nonlethal CAD events.

The choice of dose ( $1 \mathrm{~g}$ of $\mathrm{n}-3$ fatty acids containing $465 \mathrm{mg}$ EPA and $375 \mathrm{mg}$ DHA per day) and the relatively high background intake of n-3 PUFAs reported in the ORIGIN trial (the Outcome Reduction with an Initial Glargine Intervention) [64] might explain the lack of beneficial effects of n-3 PUFAs on cardiovascular mortality and morbidity in this study. This international, multicenter, randomized, and open-label trial with a $2 \times 2$ factorial design evaluated the protective effects of $n-3$ PUFAs in a daily dose of $1 \mathrm{~g}$ versus corn oil, and Insulin Glargine (Lantus) versus standard care, on cardiovascular mortality and morbidity during 6 years of followup in 12536 high-risk subjects with impaired fasting glucose, impaired glucose tolerance, or early type 2 diabetes. The estimated median dietary intake of $n-3$ PUFAs of $210 \mathrm{mg} /$ day might have muted the potential effects of $n-3$ treatment, assuming that maximal risk reduction is obtained by consuming $250 \mathrm{mg}$ n-3 PUFAs/day, as suggested by Mozaffarian and Rimm [62]. 
Several other trials have confirmed an inverse association between intake of n-3 PUFAs and risk of CAD [3, 65-70], especially fatal cardiac events $[65,67-70]$. This finding has been evident both in the setting of primary $[3,65,66,68-$ $70]$ and secondary prevention [52, 53, 67]. Only a few epidemiological studies and randomized controlled trials have presented results indicative of a lack of effect $[54,55,71-$ 74] or a direct harmful effect of a high intake of n-3 PUFAs $[75,76]$. The lack of effect on CAD from a relatively high intake of n-3 PUFAs in Western coastal populations has been suggested to be due to a concomitant high intake of saturated FAs and monounsaturated FAs [77]. The Western coastal populations differ not only from the Japanese with respect to n-3 PUFA levels; they also ingest more of the apparently unhealthy FAs with a possible attenuation of the health effects of $n-3$ PUFAs. In this setting we cannot rule out the harmful effects of environmental contaminants of fish and increases of oxidative stress, as previously discussed. The major clinical trial data for primary and secondary prevention of CVD are summarized in Table 2(a).

4.2. Studies on Antiarrhythmic Effects. Time-course analyses of the GISSI-Prevenzione study with a reduction in SCD already after 4 months of supplementation [83] have given rise to the hypothesis of a predominant antiarrhythmic effect of n-3 PUFAs. This hypothesis is supported by a case control study including 334 patients with primary cardiac arrest performed by Siscovick et al. [84] who demonstrated that an intake of $5.5 \mathrm{~g}$ of n-3 PUFAs per month (equivalent to one fatty fish meal per week) as compared to no intake was associated with a $50 \%$ reduction in the risk of cardiac arrest (95\% CI 0.4-0.8). All cases and controls were free of prior clinical heart disease. In the same study, there was an inverse relationship between blood measurements of EPA + DHA and risk of cardiac arrest. The same has been evident for SCD in the Physicians' Health Study [85] and for fatal ischemic heart disease in the Cardiovascular Health Study [86]. In the latter study, patients over the age of 65 had a $77 \%$ lower risk of assumed arrhythmic death for each standard deviation increase in plasma phospholipid DHA + EPA. None of these studies can, however, document an antiarrhythmic mechanism of protection against SCD, as the electrical activity of the myocardium at the moment of cardiac arrest was not systematically registered.

The first evidence of an antiarrhythmic potential of n3 PUFAs came from experimental work in animal models [87-90], and in a systematic review and meta-analysis of the impact of n-3 PUFAs on selected arrhythmia outcomes in these animal models, Matthan et al. [90] conclude that there is a beneficial effect of EPA and DHA on ischemiainduced ventricular fibrillation (VF) and ventricular fibrillation (VT) across all species. For ventricular arrhythmias induced by reperfusion, the results were inconsistent, and none of the animal models evaluated other arrhythmogenic mechanisms, such as scar-related malignant arrhythmias.

In attempts to determine whether $n-3$ PUFAs could have the same antiarrhythmic effects in humans, several studies have been performed in ICD patients with a high risk of recurrent ventricular arrhythmias. Schrepf et al. [45] were able to abort the inducibility of VT in 5 out of 7 ICD patients undergoing electrophysiological testing by intravenously infusing $3.8 \mathrm{~g}$ of n-3 PUFAs. The same finding was recently published by Madsen et al. [81] testing eight ICD patients undergoing a randomized, placebo-controlled, crossover study with electrophysiological testing performed both after infusion of $3.9 \mathrm{~g}$ of n-3 PUFAs and placebo. Of the 5 patients who were inducible after placebo, 2 were no longer inducible after n-3 PUFAs infusion, and another 2 required stronger stimulation to induce VT. Comparable to these findings, Christensen et al. [46] have also demonstrated that ICD patients with a low content of n-3 PUFAs in serum have a higher incidence of ventricular arrhythmias as compared to patients with high serum levels $(P<0.05)$.

Results have been less consistent in studies of orally administered supplementation of n-3 PUFAs. Leaf et al. [47] could only demonstrate a trend toward prolonged time to the first ICD event (VF or VT) for patients receiving $4 \mathrm{~g} /$ day of fish oil supplements (total dose of EPA and DHA of $2.6 \mathrm{~g} /$ day) as compared to olive oil supplements for 12 months, while Metcalf et al. [44] found that a daily dose of $3 \mathrm{~g}$ encapsulated fish oil for approximately 6 weeks resulted in noninducible or less inducible VT in a group of patients with ischemic cardiomyopathy. Brouwer and colleagues [78] found no strong evidence for a protective effect of a daily dose of $0.9 \mathrm{~g} \mathrm{n}-3$ PUFAs for 1 year as compared to that of sunflower oil in 546 ICD patients. A similar dose $(1 \mathrm{~g} /$ day of n-3 PUFAs) did, however, result in a trend toward protection in a substudy of the GISSI-HF trial when given to 566 patients for a median followup duration of 928 days [79]. There was a nonsignificant $20 \%$ reduction in appropriate ICD managed VT/VF events in the n-3 PUFA group as compared to placebo. Interestingly, this did not result in any mortality benefit. There was actually a minimal excess in total mortality observed in the group treated with n-3 PUFAs $(26.6 \%$ versus $24.3 \%)$. This is in some contrast to the results of the main study where the greatest proportion of the absolute risk reduction of total mortality by $n-3$ PUFA supplementation was attributable to a reduction in presumed arrhythmic deaths [56]. Moreover, in the study by Raitt et al. [80], recurrent episodes of VT or VF during 2 years of followup occurred more frequently and with reduced time to event in patients receiving $1.3 \mathrm{~g}$ /day of EPA/DHA as compared to olive oil.

As previously mentioned, the protective effect of $n-3$ PUFAs might be strongest for ischemia-induced ventricular arrhythmias. The implantation of an ICD is more often performed in the setting of VF or VT without a concomitant MI or any reversible cause or in the case of high risk of SCD, such as excessive heart failure or demonstration of inducible VF/VT at electrophysiological examination. Even though ischemia might contribute to recurrent arrhythmic events, these patients may also have other arrhythmic substrates which to a lesser degree may be affected by n-3 PUFAs. The mixture of both VF and VT as a primary endpoint in most of the ICD studies also complicates the comparisons. The study by Christensen et al. [46] demonstrating a significantly lower frequency of ICD events for patients with 


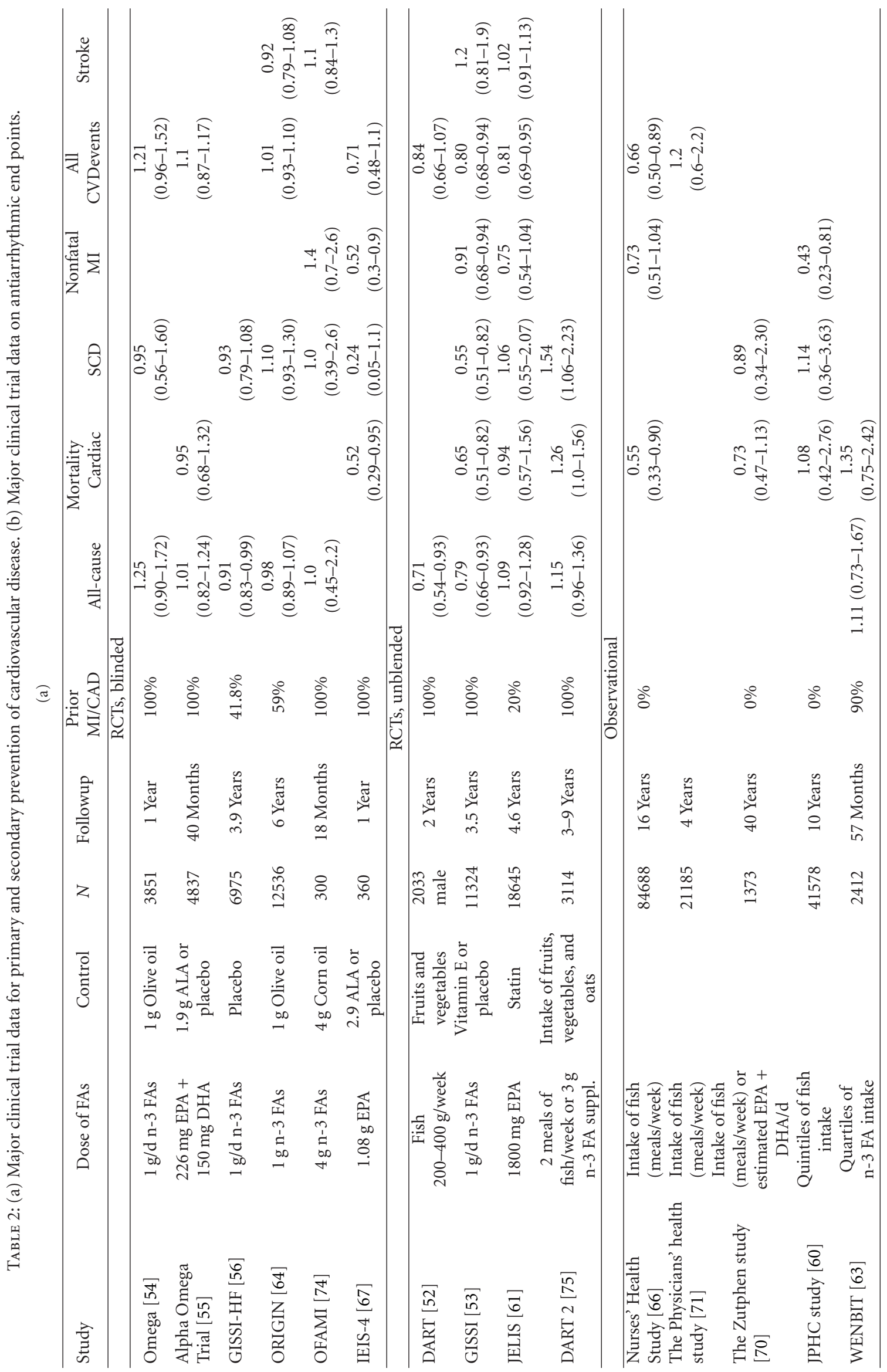




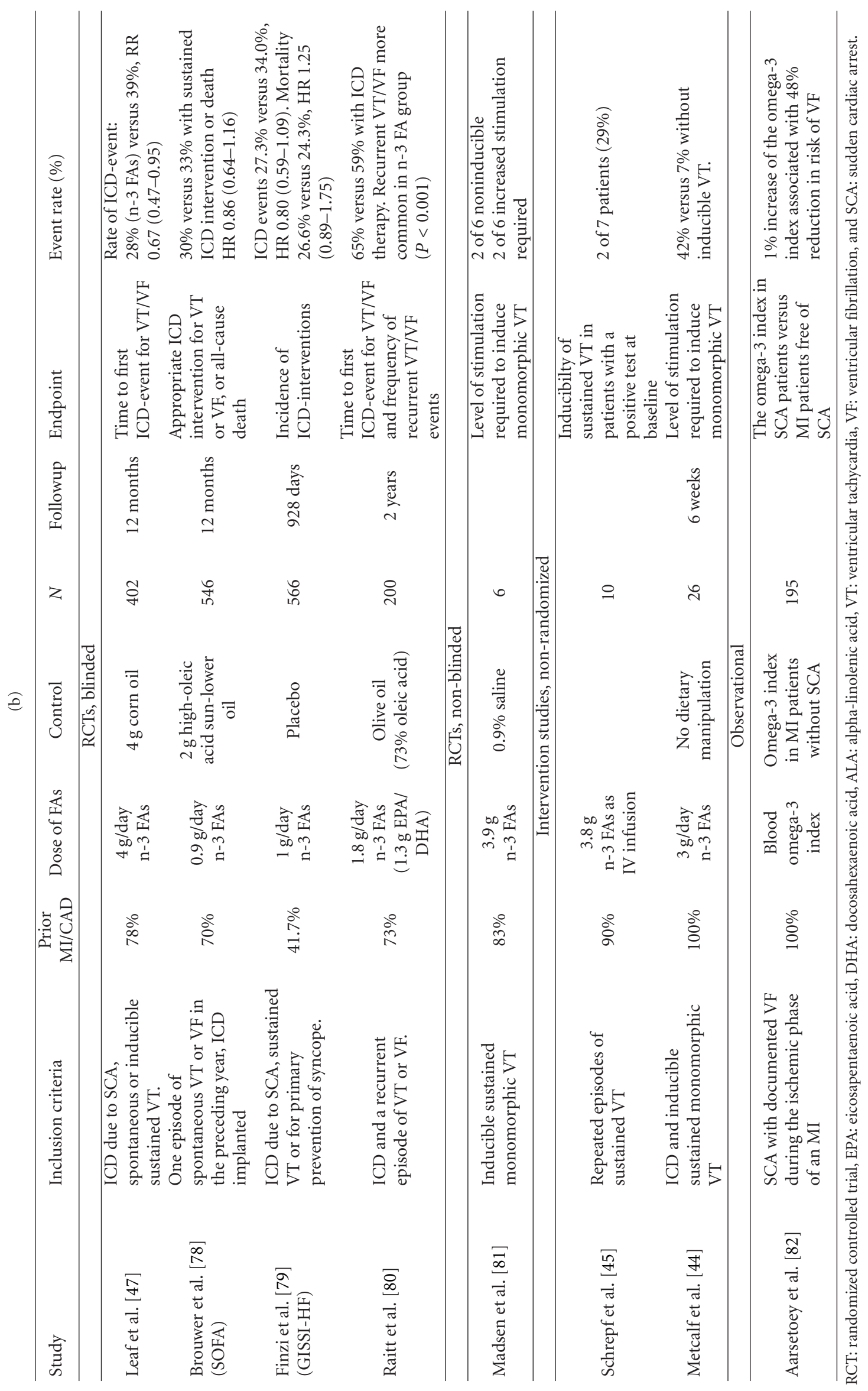


the highest serum content of n-3 PUFAs reported only a reduction in the presence of VF. Even though a reduction in inducible VT has been achieved in both animals and humans after infusion of n-3 PUFAs as well as after highdose supplementation [44], it is not known whether these marine PUFAs in normal dietary or supplementary ingested doses have the same potential to reduce the occurrence of spontaneous VT as compared to VF. It is, however, interesting to note that those studies reporting no beneficial effect of oral supplementation seem to have the highest proportion of patients with only VT prior to inclusion $[78,80]$. Furthermore, there is a high diversity between the ICD studies in supplementation doses and the length of the intervention, making an overall conclusion even harder to achieve.

In agreement with a possible antiarrhythmic effect in the setting of ischemia-induced ventricular arrhythmias, we have recently presented evidence for a reduced risk of VF during the acute ischemic phase of an MI with high levels of cellular EPA and DHA [91]. After adjustment for other potential predictors of risk of VF, our case-control study including 10 patients with VF during the initial 6 hours of symptom onset suggested a $48 \%$ reduction in risk of this life-threatening arrhythmia with a $1 \%$ increase of the omega-3 index. We have later been able to reproduce the same finding in another SCD population comprising 12 case patients with a first-time MI [82]. The main strength of both of these studies is the documentation of VF at the time of cardiac arrest, lending support to the hypothesis of an antiarrhythmic effect of n-3 PUFAs. Interestingly, results of the electrophysiological studies performed both in animals [88] and humans [45, 81 ], demonstrating reduced inducibility or termination of ventricular arrhythmias immediately after IV infusion of n-3 PUFAs, also suggest that incorporation of marine PUFAs into cell membranes might not be necessary for their antiarrhythmic effect. The major clinical trial data on antiarrhythmic effects are given in Table 2(b).

4.3. Meta-Analyses. A meta-analysis by Hooper et al. published in Britical Medical Journal (BMJ) in 2006 was given a lot of attention in media due to the conclusion that $n-3$ PUFAs given for at least 6 months had no clear effect on total mortality or combined CVD events [92]. This analysis was based on 41 cohort studies and 48 randomized intervention trials including both patients with and without established CVD but has later been criticized for several methodological problems. A recently published meta-analysis by Filion et al. [93] comprising 29 randomized controlled trials (RCTs) including 35144 high-risk CVD patients could neither demonstrate a statistically significant decrease in total mortality (RR $0.88,95 \%$ CI $0.64-1.03$ ) nor an effect on restenosis prevention (RR $0.89,95 \%$ CI $0.72-1.06$ ) by n-3 PUFAs.

The results of these reviews differ, however, from several other meta-analyses evaluating observational studies and RCTs [94-99]. They all demonstrate a reduced risk of cardiac death with increasing intake of marine PUFAs. The review by $\mathrm{He}$ et al. [95] could actually demonstrate a 7\% lower risk of coronary heart disease mortality for each $20 \mathrm{~g} /$ day increase in fish intake. The results are less consistent when evaluating the effect of n-3 PUFAs on nonfatal CVD events. Yzebe and Lievre [96], including 10 RCTs comprising 14 727 patients in their analysis, found no significant effects on nonfatal MI, nonfatal stroke, or the presence of angina pectoris. The same lack of influence by n-3 PUFAs on the incidence of nonfatal MI was observed by Bucher et al. [99]. This is opposed to the outcome from the review of 11 RCTs including 39044 patients performed by Marik and Varon [97] where dietary supplementation with n-3 PUFAs significantly reduced the risk of nonfatal CVD events (OR $0.92, P=0.02$ ). The latter finding might, however, be due to the fact that $48 \%$ of the patients included in that metaanalysis belonged to the JELIS study [61]. Furthermore, the heterogeneity of the effect of n-3 PUFA intake on CVD outcome might be related to the impact of varying doses and time of followup.

In a meta-analysis by Kwak et al. [100] involving 20485 patients with a history of cardiovascular disease from 14 randomized, double-blind, and placebo-controlled studies, intervention with EPA and DHA showed insufficient evidence of a secondary preventive effect of n-3 PUFA supplements against overall cardiovascular events. JELIS [61] and GISSI 4 [53] were excluded from this analyses, as these were open studies.

The reduced risk of cardiac death associated with the intake of n-3 PUFAs has largely been attributed to the prevention of SCD. Two meta-analyses have specifically evaluated this outcome. Both Marik and Varon [97] and Chen et al. [98] found a reduced risk of SCD to be associated with the intake of n-3 PUFAs. In the study by Chen et al., this effect was, however, limited to CVD patients without guideline-adjusted therapy (RR 0.64, 95\% CI 0.51-0.80).

There are only a couple of meta-analyses available regarding the effect of n-3 PUFAs in ICD patients [43, 101], both of which include the previously described studies by Leaf et al. [47], Brouwer et al. [78], and Raitt et al. [80]. None of these studies support a protective effect of n-3 PUFAs from fish oil on cardiac arrhythmia in patients with an ICD.

However, the difficulties in interpreting meta-analyses are clearly demonstrated by the recently published two meta-analyses by Rizos et al. [102] and Delgado-Lista et al. [103] evaluating the efficacy of omega-3 PUFAs on CVD, including 12 of the same studies, but drawing opposite conclusions. Both include randomized controlled trials in primary and secondary prevention, but the endpoints differ. Rizos et al. [102], including 20 studies, conclude that omega3 PUFA supplementation was not associated with a lower risk of all-cause mortality, cardiac death, sudden death, myocardial infarction, or stroke, based on relative and absolute measures of association. On the contrary, DelgadoLista et al. [103], including 21 studies, conclude in favour of omega-3 fatty acids, stating that marine omega- 3 fatty acids are effective in preventing cardiovascular events of any kind (composite endpoint of stroke, coronary events, myocardial infarction or angina pectoris, peripheral limb disease events, or death from cardiovascular causes), cardiac death, and coronary events, especially in persons with high cardiovascular risk. 
4.4. Studies on Triglycerides. The triglyceride-lowering effect of marine n-3 PUFAs is well recognized and has been demonstrated to be linearly dose-dependent across a wide range of consumption. As early as in 1990, Schmidt et al. performed their dose-response studies on the effect of marine n-3 PUFAs on triglyceride levels, showing that 6 weeks of supplementation with $1.3,4$, and 9 grams of n3 PUFAs daily to healthy normolipidemic men resulted in a reduction in plasma triglycerides by $9 \%, 25 \%$, and $33 \%$, respectively, in response to increasing doses of n-3 PUFAs [33]

The minimal effective dose of marine n-3 PUFAs has been demonstrated to be about 1 gram daily, also in accordance with observations in the GISSI study, where $1 \mathrm{~g}$ marine n-3 PUFAs for 6 months resulted in a small, but significant, triglyceride reduction [53].

Even greater dose-dependent reductions in plasma levels of triglycerides of $40 \%-50 \%$ have been observed among individuals with hypertriglyceridemia. The American Heart Association (AHA) [104] recommendations state that EPADHA supplements may be useful in patients with severe hypertriglyceridemia ( $>500 \mathrm{mg}$ of triglycerides per deciliter ( $5.6 \mathrm{mmol}$ per liter)) in doses of 2 to $4 \mathrm{~g}$ of EPA-DHA per day to lower triglyceride levels by $20 \%$ to $40 \%$ in people in whom diet and lifestyle measures have not led to appropriate concentrations of triglycerides. This is also in agreement with the US National Cholesterol Education Program (NCEP) [105]. However, a meta-analysis studying the triglyceride-lowering effect of marine n-3 PUFAs in a daily dose of 4 grams administered to subjects with moderate hypertriglyceridemia (triglycerides $150-500 \mathrm{mg} / \mathrm{dL}$ ) concludes that marine $\mathrm{n}-3$ PUFAs are effective in reducing triglycerides by approximately 30\% [106]. Additional lipid disturbances and CVD risk factors should be considered before therapeutic decisions are made. Although clinical studies have reported considerable triglyceride-lowering effects by marine n-3 PUFA supplementation, no data for clinical endpoints are available to lend support to this recommendation. Further investigation is needed to explore this area.

\section{The Omega-3 Index-A New Risk Factor for CAD?}

The omega-3 index has during the recent decade been proposed as a new risk factor for CVD, especially for fatal cardiac events $[48,107,108]$. This index is an independent measurement of the amount of EPA and DHA available in the body, highly reflecting the FA composition of the myocardium [35-37]. In case-control studies the omega3 index has been demonstrated to be an independent risk marker for SCD [84] and for the development of an ACS [109, 110].

We could, however, not demonstrate any prognostic utility of the omega-3 index in the setting of secondary prevention in 460 ACS patients from the "Risk Markers in the Acute Coronary Syndrome study” (RACS) [111] with respect to future cardiac events or risk of death after adjustment for traditional risk factors and established risk markers. We have, however, in two different populations of first-time MIpatients demonstrated low levels of the omega- 3 index to be independently associated with increased risk of cardiac arrest/VF during the acute ischemic phase of an MI $[82,91]$.

The omega-3 index appears to fulfill many of the criteria required for a risk marker/risk factor, especially for SCD $[48,107,108]$. It has been estimated that the highest risk of cardiac death is associated with an omega- 3 index below $4 \%$, with a level of $8 \%$ offering the greatest degree of cardioprotection $[48,108]$. These estimates correspond well with the actual measurements in our cardiac arrest patients but could not be confirmed as cut-points to classify patients at low, intermediate, or high risk in our prognostic study. Further studies are needed to elucidate the final role of the omega-3 index in a clinical setting.

\section{Conclusions and Further Perspectives}

Despite a significant amount of research since the pioneering work of Bang and Dyerberg in the 1970s attempting to elucidate the possible health effects of n-3 PUFAs, there are still several areas of uncertainty. The existing literature is, however, mainly supportive of a cardioprotective effect of marine fish oils, even though there is some disagreement as to whether this effect is mediated through a reduced risk of fatal or nonfatal cardiac disease. This might, however, be a question of an optimum dose or whether there exists a doseresponse relation for n-3 PUFA supplementation. A final conclusion is difficult to reach, as studies differ in design and are performed in highly different patient populations with different background intake of fish and reflect the administration of a wide range of supplement doses of $n$ 3 PUFAs for varying periods of time. We definitely need more high quality, large-scale, randomized, and controlled clinical trials of long-term duration also reporting possible harmful effects. Furthermore, studies on out-of-hospital cardiac arrest will hopefully answer some more questions related to the association between n-3 PUFAs and risk of SCD. Moreover, to diminish difficulties in interpreting meta-analyses, future meta-analyses should strive to include trials with more homogenous designs and populations with respect to cardiovascular risk profile, using similar doses of marine n-3 PUFAs, with precisely defined endpoints.

We are still awaiting results of three large-scaled trials which might contribute to a further understanding of the appropriate role of $n-3$ PUFAs in the primary prevention of cardiovascular events in both high- and low-risk participants. In the large ASCEND (A Study of Cardiovascular Events in Diabetes) trial, 15480 diabetic patients (type I and II) without CVD at the time of inclusion were randomized with a $2 \times 2$ factorial design to receive low-dose aspirin (100 mg), n-3 PUFA $1 \mathrm{~g} /$ day, both regimens or placebo in a $2 \times 2$. Followup is scheduled to continue until 2017. No publication is yet available. The Rischio and Prevenzione study has between 2004 and 2007 randomized 12513 participants without a previous MI to a daily dose of $1 \mathrm{~g}$ of n-3 PUFAs or placebo (olive oil) for 5 years to evaluate the influence of $n-3$ PUFAs on death or hospitalization 
TABLE 3: Official recommendations for the use of marine n-3 fatty acids in CVD prevention.

\begin{tabular}{ll}
\hline & Primary prevention \\
\hline & \\
& $\begin{array}{l}\text { Eat a variety of fish, preferably oily fish (salmon, } \\
\text { tuna, mackerel, herring, and trout), at least twice a } \\
\text { Theek. Consuming fish oil supplements should only } \\
\text { be considered by people with high levels of } \\
\text { triglycerides who consult with their physicians. }\end{array}$
\end{tabular}

Secondary prevention

Consume about 1 gram per day of the fish oils EPA and DHA (eicosapentaenoic and docosahexaenoic acids), preferably from oily fish, although EPA + DHA supplements could be considered in consultation with their physicians.

People who have elevated triglycerides may need two to four grams of EPA and DHA per day provided as capsules under a physician's care.

Eat fish twice a week, of which once oily fish ${ }^{2}$. The recommended doses of total EPA and DHA to

European Society of lower triglycerides have varied between 2 and Eat fish twice a week, of which once oily fish ${ }^{2}$. The recommended doses of total EPA and DHA to Cardiology (ESC) $4 \mathrm{~g} /$ day. Use of n-3 fatty acids (prescription lower triglycerides have varied between 2 and $4 \mathrm{~g} /$ day. Use of n-3 fatty acids (prescription products) as an adjunct to the diet if triglycerides exceed $5.6 \mathrm{mmol} / \mathrm{L}(496 \mathrm{mg} / \mathrm{dL})^{3}$. products) as an adjunct to the diet if triglycerides exceed $5.6 \mathrm{mmol} / \mathrm{L}(496 \mathrm{mg} / \mathrm{dL})^{3}$.

Encourages increased consumption of omega- 3 fatty acids in the form of fish or in capsule form $(1 \mathrm{~g} / \mathrm{d})$

American College of Cardiology (ACC) No recommendation. for risk reduction. For treatment of elevated triglycerides, higher doses are usually necessary for risk reduction ${ }^{4}$.

\begin{tabular}{ll}
\hline $\begin{array}{l}\text { International Society for } \\
\text { the Study of Fatty Acids } \\
\text { and Lipids (ISSFAL) }\end{array}$ & $\begin{array}{l}\text { A minimum intake of eicosapentaenoic acid (EPA) } \\
\text { and docosahexaenoic acid (DHA) combined, of } \\
500 \mathrm{mg} / \mathrm{d} \text {. }\end{array}$ \\
\hline $\begin{array}{l}\text { Scientific Advisory } \\
\text { Committee on }\end{array}$ & $\begin{array}{l}\text { Recommends the equivalent of } 450 \mathrm{mg} \text { marine } \\
\text { omega-3 daily and an increase in population oily fish } \\
\text { Nutrition, United }\end{array}$ \\
$\begin{array}{l}\text { Kingdom } \\
\text { consumption to one portion a week. }\end{array}$
\end{tabular}

No recommendation.

Recommends the equivalent of $450 \mathrm{mg}$ marine omega-3 daily and an increase in population oily fish consumption to one portion a week.

$(\mathrm{UK} \text { SACN })^{6}$

World Health Organization $(\mathrm{WHO})^{7}$
Regular fish consumption (1-2 servings per week) is protective against coronary heart disease and ischaemic stroke and is recommended. The serving should provide an equivalent of 200-500 mg of eicosapentaenoic and docosahexaenoic acid.
Regular fish consumption (1-2 servings per week) is protective against coronary heart disease and ischaemic stroke and is recommended. The serving should provide an equivalent of 200-500 mg of eicosapentaenoic and docosahexaenoic acid.

\footnotetext{
${ }^{1}$ http://www.heart.org/.

${ }^{2}$ European Guidelines on cardiovascular disease prevention in clincal practice (version 2012). The fifth joint task.

Force of the European Society of Cardiology and Other Societies on Cardiovascular Disease Prevention in Clinical Practice. EHJ 2012; 33: 1635-1701. (ESC) and the European Atherosclerosis Society (EAS). EHJ 2011; 32(14): 1769-1818. National Heart, Lung, and Blood Institute. JACC 2006; 47(10): 2130-9.

${ }^{5}$ http://www.issfal.org/.

${ }^{6}$ Advice on Fish Consumption: Benefits and Risks was published in 2004 by the joint SACN/COT Subgroup (SACN 2004).

${ }^{7}$ World Health Organization (WHO) 2003: "Diet, Nutrition and the Prevention of Chronic Diseases."
}

${ }^{3}$ ESC/EAS Guidelines for the management of dyslipidaemia. The Task Force for the management of dyslipidaemias of the European Society of Cardiology

${ }^{4}$ AHA/ACC Guidelines for Secondary Prevention for Patients with Coronary and Other Atherosclerotic Vascular Disease: 2006 update endorsed by the

for cardiovascular events. Finally, the third large study, the Vitamin D and Omega-3 Trial (VITAL), is currently recruiting 20000 participants in the US without a history of CVD and cancer, for supplementation with Vitamin D and/or $1 \mathrm{~g} \mathrm{n}$-3 PUFAs, to assess the preventive effects of n3 PUFAs on these conditions.

The official recommendations for the use of marine n3 fatty acids in CVD prevention by major official organizations such as The American Heart Association (AHA), the European Society of Cardiology (ESC), the International Society for the Study of Fatty Acids and Lipids (ISSFAL), and the World Health Organization (WHO) are given in Table 3. However, based on the existing knowledge, the general recommendation of fish or fish oil supplements in patients at risk of, or with established CVD, has not been substantiated, but high doses of n-3 PUFAs appear to be safe in combination with current recommendations regarding medical treatment of $\mathrm{CAD}$ patients, especially when combined with aspirin and clopidogrel [112].

\section{References}

[1] H. O. Bang and J. Dyerberg, "Lipid metabolism and ischemic heart disease in Greenland Eskimos," in Advanced Nutrition Research, H. H. Draper, Ed., vol. 3, pp. 1-32, Plenum Press, New York, NY, USA, 1980.

[2] P. Libby, "Coronary artery injury and the biology of atherosclerosis: inflammation, thrombosis, and stabilization," American Journal of Cardiology, vol. 86, no. 8, pp. 3J-9J, 2000.

[3] T. Rissanen, S. Voutilainen, K. Nyyssonen, T. A. Lakka, and J. T. Salonen, "Fish oil-derived fatty acids, docosahexaenoic 
acid and docosapentaenoic acid, and the risk of acute coronary events: The Kuopio Ischaemic Heart Disease Risk Factor Study," Circulation, vol. 102, no. 22, pp. 2677-2679, 2000.

[4] E. Guallar, M. I. Sanz-Gallardo, P. van't Veer et al., "Mercury, fish oils, and the risk of myocardial infarction," The New England Journal of Medicine, vol. 347, no. 22, pp. 1747-1754, 2002.

[5] J. T. Salonen, K. Seppänen, T. A. Lakka, R. Salonen, and G. A. Kaplan, "Mercury accumulation and accelerated progression of carotid atherosclerosis: a population-based prospective 4year follow-up study in men in eastern Finland," Atherosclerosis, vol. 148, no. 2, pp. 265-273, 2000.

[6] H. Grundt, D. W. T. Nilsen, M. A. Mansoor, and A. Nordøy, "Increased lipid peroxidation during long-term intervention with high doses of n-3 fatty acids (PUFAs) following an acute myocardial infarction," European Journal of Clinical Nutrition, vol. 57, no. 6, pp. 793-800, 2003.

[7] C. A. Drevon, "Marine oils and their effects," Nutrition Reviews, vol. 50, pp. 29-36, 1992.

[8] D. Mozaffarian and J. H. Y. Wu, "Omega-3 fatty acids and cardiovascular disease. Effects on risk factors, molecular pathways, and clinical events," Journal of the American College of Cardiology, vol. 58, no. 20, pp. 2047-2067, 2011.

[9] A. Leaf, Y. F. Xiao, J. X. Kang, and G. E. Billman, "Prevention of sudden cardiac death by n-3 polyunsaturated fatty acids," Pharmacology and Therapeutics, vol. 98, no. 3, pp. 355-377, 2003.

[10] R. A. Siddiqui, K. A. Harvey, and G. P. Zaloga, "Modulation of enzymatic activities by n-3 polyunsaturated fatty acids to support cardiovascular health," The Journal of Nutritional Biochemistry, vol. 19, no. 7, pp. 417-437, 2008.

[11] H. Li, X. Z. Ruan, S. H. Powis et al., "EPA and DHA reduce LPS-induced inflammation responses in HK-2 cells: evidence for a PPAR- $\gamma$-dependent mechanism," Kidney International, vol. 67, no. 3, pp. 867-874, 2005.

[12] C. Monaco, E. Andreakos, S. Kiriakidis et al., "Canonical pathway of nuclear factor $\kappa \mathrm{B}$ activation selectively regulates proinflammatory and prothrombotic responses in human atherosclerosis," Proceedings of the National Academy of Sciences of the United States of America, vol. 101, no. 15, pp. 5634-5639, 2004.

[13] S. A. Khan and J. P. Vanden Heuvel, "Reviews: current topics role of nuclear receptors in the regulation of gene expression by dietary fatty acids (review)," The Journal of Nutritional Biochemistry, vol. 14, no. 10, pp. 554-567, 2003.

[14] R. De Caterina, "Drug therapy: n-3 fatty acids in cardiovascular disease," The New England Journal of Medicine, vol. 364, no. 25, pp. 2439-2450, 2011.

[15] P. Saravanan, N. C. Davidson, E. B. Schmidt, and P. C. Calder, "Cardiovascular effects of marine omega-3 fatty acids," The Lancet, vol. 376, no. 9740, pp. 540-550, 2010.

[16] J. G. Robinson and N. J. Stone, "Antiatherosclerotic and antithrombotic effects of omega-3 fatty acids," American Journal of Cardiology, vol. 98, no. 4, pp. i39-i49, 2006.

[17] J. A. Reiffel and A. McDonald, "Antiarrhythmic effects of omega-3 fatty acids," American Journal of Cardiology, vol. 98, no. 4, pp. i50-i60, 2006.

[18] W. P. Newman, J. P. Middaugh, M. T. Propst, and D. R. Rogers, "Atherosclerosis in Alaska natives and non-natives," The Lancet, vol. 341, no. 8852, pp. 1056-1057, 1993.

[19] J. H. Rapp, W. E. Connor, D. S. Lin, and J. M. Porter, "Dietary eicosapentaenoic acid and docosahexaenoic acid from fish oil: their incorporation into advanced human atherosclerotic plaques," Arteriosclerosis and Thrombosis, vol. 11, no. 4, pp. 903-911, 1991.

[20] F. Thies, J. M. C. Garry, P. Yaqoob et al., "Association of n3 polyunsaturated fatty acids with stability of atherosclerotic plaques: a randomised controlled trial," The Lancet, vol. 361, no. 9356, pp. 477-485, 2003.

[21] C. von Schacky, P. Angerer, W. Kothny, K. Theisen, and H. Mudra, "The effect of dietary $\omega-3$ fatty acids on coronary atherosclerosis. A randomized, double-blind, placebocontrolled trial," Annals of Internal Medicine, vol. 130, no. 7, pp. 554-562, 1999.

[22] F. M. Sacks, F. H. Stone, C. M. Gibson, D. I. Silverman, B. Rosner, and R. C. Pasternak, "Controlled trial of fish oil for regression of human coronary atherosclerosis," Journal of the American College of Cardiology, vol. 25, no. 7, pp. 1492-1498, 1995.

[23] A. T. Erkkilä, N. R. Matthan, D. M. Herrington, and A. H. Lichtenstein, "Higher plasma docosahexaenoic acid is associated with reduced progression of coronary atherosclerosis in women with CAD," Journal of Lipid Research, vol. 47, no. 12, pp. 2814-2819, 2006.

[24] H. O. Bang, J. Dyerberg, and A. B. Nielsen, "Plasma lipid and lipoprotein pattern in Greenlandic West-coast Eskimos," The Lancet, vol. 1, no. 7710, pp. 1143-1145, 1971.

[25] K. H. Bonaa, K. S. Bjerve, B. Straume, I. T. Gram, and D. Thelle, "Effect of eicosapentaenoic and docosahexaenoic acids on blood pressure in hypertension. A population-based intervention trial from the Tromso study," The New England Journal of Medicine, vol. 322, no. 12, pp. 795-801, 1990.

[26] J. Luo, S. W. Rizkalla, J. Boillot et al., "Dietary (n-3) polyunsaturated fatty acids improve adipocyte insulin action and glucose metabolism in insulin-resistant rats: relation to membrane fatty acids," The Journal of Nutrition, vol. 126, no. 8, pp. 1951-1958, 1996.

[27] A. Chicco, M. E. D’Alessandro, L. Karabatas, R. Gutman, and Y. B. Lombardo, "Effect of moderate levels of dietary fish oil on insulin secretion and sensitivity, and pancreas insulin content in normal rats," Annals of Nutrition and Metabolism, vol. 40, no. 2, pp. 61-70, 1996.

[28] G. K. Hansson, P. Libby, U. Schönbeck, and Z. Q. Yan, "Innate and adaptive immunity in the pathogenesis of atherosclerosis," Circulation Research, vol. 91, no. 4, pp. 281291, 2002.

[29] J. Dyerberg and H. O. Bang, "Haemostatic function and platelet polyunsaturated fatty acids in Eskimos," The Lancet, vol. 2, no. 8140, pp. 433-435, 1979.

[30] H. O. Bang and J. Dyerberg, "The bleeding tendency in Greenland Eskimos," Danish Medical Bulletin, vol. 27, no. 4, pp. 202-205, 1980.

[31] W. S. Harris, M. Miller, A. P. Tighe, M. H. Davidson, and E. J. Schaefer, "Omega-3 fatty acids and coronary heart disease risk: clinical and mechanistic perspectives," Atherosclerosis, vol. 197, no. 1, pp. 12-24, 2008.

[32] H. Grundt, D. W. T. Nilsen, M. A. Mansoor, Ø. Hetland, and A. Nordøy, "Reduction in homocysteine by n-3 polyunsaturated fatty acids after 1 year in a randomised doubleblind study following an acute myocardial infarction: no effect on endothelial adhesion properties," Pathophysiology of Haemostasis and Thrombosis, vol. 33, no. 2, pp. 88-95, 2003.

[33] E. B. Schmidt, K. Varming, E. Ernst, P. Madsen, and J. Dyerberg, "Dose-response studies on the effect of n-3 polyunsaturated fatty acids on lipids and haemostasis," Thrombosis and Haemostasis, vol. 63, no. 1, pp. 1-5, 1990. 
[34] J. Eritsland, H. Arnesen, I. Seljeflot, and P. Kierulf, "Longterm effects of n-3 polyunsaturated fatty acids on haemostatic variables and bleeding episodes in patients with coronary artery disease," Blood Coagulation and Fibrinolysis, vol. 6, no. 1, pp. 17-22, 1995.

[35] W. S. Harris, S. A. Sands, S. L. Windsor et al., "Omega3 fatty acids in cardiac biopsies from heart transplantation patients: correlation with erythrocytes and response to supplementation," Circulation, vol. 110, no. 12, pp. 16451649, 2004.

[36] A. J. Owen, B. A. Peter-Przyborowska, A. J. Hoy, and P. L. McLennan, "Dietary fish oil dose- and time-response effects on cardiac phospholipid fatty acid composition," Lipids, vol. 39, no. 10, pp. 955-961, 2004.

[37] M. L. Garg, J. Leitch, R. J. Blake, and R. Garg, "Long-chain n-3 polyunsaturated fatty acid incorporation into human atrium following fish oil supplementation," Lipids, vol. 41, no. 12, pp. 1127-1132, 2006.

[38] S. Nodari, M. Metra, G. Milesi et al., "The role of n-3 PUFAs in preventing the arrhythmic risk in patients with idiopathic dilated cardiomyopathy," Cardiovascular Drugs and Therapy, vol. 23, no. 1, pp. 5-15, 2009.

[39] D. Mozaffarian, A. Geelen, I. A. Brouwer, J. M. Geleijnse, P. L. Zock, and M. B. Katan, "Effect of fish oil on heart rate in humans: a meta-analysis of randomized controlled trials," Circulation, vol. 112, no. 13, pp. 1945-1952, 2005.

[40] D. Mozaffarian, P. K. Stein, R. J. Prineas, and D. S. Siscovick, "Dietary fish and $\omega-3$ fatty acid consumption and heart rate variability in US adults," Circulation, vol. 117, no. 9, pp. 1130-1137, 2008.

[41] X. Jouven, M. Zureik, M. Desnos, C. Guérot, and P. Ducimetière, "Resting heart rate as a predictive risk factor for sudden death in middle-aged men," Cardiovascular Research, vol. 50, no. 2, pp. 373-378, 2001.

[42] N. R. Matthan, H. Jordan, M. Chung, A. H. Lichtenstein, D. A. Lathrop, and J. Lau, "A systematic review and metaanalysis of the impact of $\omega-3$ fatty acids on selected arrhythmia outcomes in animal models," Metabolism, vol. 54, no. 12, pp. 1557-1565, 2005.

[43] I. A. Brouwer, M. H. Raitt, C. Dullemeijer et al., "Effect of fish oil on ventricular tachyarrhythmia in three studies in patients with implantable cardioverter defibrillators," European Heart Journal, vol. 30, no. 7, pp. 820-826, 2009.

[44] R. G. Metcalf, P. Sanders, M. J. James, L. G. Cleland, and G. D. Young, "Effect of dietary n-3 polyunsaturated fatty acids on the inducibility of ventricular tachycardia in patients with ischemic cardiomyopathy," American Journal of Cardiology, vol. 101, no. 6, pp. 758-761, 2008.

[45] R. Schrepf, T. Limmert, P. C. Weber, K. Theisen, and A. Sellmayer, "Immediate effects of n-3 fatty acid infusion on the induction of sustained ventricular tachycardia," The Lancet, vol. 363, no. 9419, pp. 1441-1442, 2004.

[46] J. H. Christensen, S. Riahi, E. B. Schmidt et al., "N-3 fatty acids and ventricular arrhythmias in patients with ischaemic heart disease and implantable cardioverter defibrillators," Europace, vol. 7, no. 4, pp. 338-344, 2005.

[47] A. Leaf, C. M. Albert, M. Josephson et al., "Prevention of fatal arrhythmias in high-risk subjects by fish oil n-3 fatty acid intake," Circulation, vol. 112, no. 18, pp. 2762-2768, 2005.

[48] W. S. Harris and C. Von Schacky, “The omega-3 index: a new risk factor for death from coronary heart disease?" Preventive Medicine, vol. 39, no. 1, pp. 212-220, 2004.
[49] Q. Sun, J. Ma, H. Campos, S. E. Hankinson, and F. B. $\mathrm{Hu}$, "Comparison between plasma and erythrocyte fatty acid content as biomarkers of fatty acid intake in US women," The American Journal of Clinical Nutrition, vol. 86, no. 1, pp. 7481, 2007.

[50] C. von Schacky and W. S. Harris, "Cardiovascular benefits of omega-3 fatty acids," Cardiovascular Research, vol. 73, no. 2, pp. 310-315, 2007.

[51] J. Cao, K. A. Schwichtenberg, N. Q. Hanson, and M. Y. Tsai, "Incorporation and clearance of omega-3 fatty acids in erythrocyte membranes and plasma phospholipids," Clinical Chemistry, vol. 52, no. 12, pp. 2265-2272, 2006.

[52] M. L. Burr, A. M. Fehily, J. F. Gilbert et al., "Effects of changes in fat, fish, and fibre intakes on death and myocardial reinfarction: Diet and reinfarction trial (DART)," The Lancet, vol. 2, no. 8666, pp. 757-761, 1989.

[53] R. Marchioli, "Dietary supplementation with N-3 polyunsaturated fatty acids and vitamin $\mathrm{E}$ after myocardial infarction: results of the GISSI-Prevenzione trial," The Lancet, vol. 354, no. 9177, pp. 447-455, 1999.

[54] B. Rauch, R. Schiele, S. Schneider et al., "OMEGA, a randomized, placebo-controlled trial to test the effect of highly purified omega-3 fatty acids on top of modern guidelineadjusted therapy after myocardial infarction," Circulation, vol. 122, no. 21, pp. 2152-2159, 2010.

[55] D. Kromhout, E. J. Giltay, and J. M. Geleijnse, "N-3 fatty acids and cardiovascular events after myocardial infarction," The New England Journal of Medicine, vol. 363, no. 21, pp. 20152026, 2010.

[56] Gissi-HF Investigators, L. Tavazzi, A. P. Maggioni et al., "Effect of n-3 polyunsaturated fatty acids in patients with chronic heart failure (the GISSI-HF trial): a randomised, double-blind, placebo-controlled trial," The Lancet, vol. 372, pp. 1223-1230, 2008.

[57] S. Ghio, L. Scelsi, R. Latini et al., "Effects of n-3 polyunsaturated fatty acids and of rosuvastatin on left ventricular function in chronic heart failure: a substudy of GISSI-HF trial," European Journal of Heart Failure, vol. 12, no. 12, pp. 1345-1353, 2010.

[58] D. Moertl, A. Hammer, S. Steiner, R. Hutuleac, K. Vonbank, and R. Berger, "Dose-dependent effects of omega3-polyunsaturated fatty acids on systolic left ventricular function, endothelial function, and markers of inflammation in chronic heart failure of nonischemic origin: a doubleblind, placebo-controlled, 3-arm study," American Heart Journal, vol. 161, no. 5, pp. 915.e1-915.e9, 2011.

[59] S. Nodari, M. Triggiani, U. Campia et al., "Effects of n-3 polyunsaturated fatty acids on left ventricular function and functional capacity in patients with dilated cardiomyopathy," Journal of the American College of Cardiology, vol. 57, no. 7, pp. 870-879, 2011.

[60] H. Iso, M. Kobayashi, J. Ishihara et al., "Intake of fish and n3 fatty acids and risk of coronary heart disease among Japanese: The Japan Public Health Center-Based (JPHC) study cohort I," Circulation, vol. 113, no. 2, pp. 195-202, 2006.

[61] M. Yokoyama, H. Origasa, M. Matsuzaki et al., "Effects of eicosapentaenoic acid on major coronary events in hypercholesterolaemic patients (JELIS): a randomised openlabel, blinded endpoint analysis," The Lancet, vol. 369, no. 9567, pp. 1090-1098, 2007.

[62] D. Mozaffarian and E. B. Rimm, "Fish intake, contaminants and human health. Evaluating the risks and benefits," Journal 
of the American Medical Association, vol. 296, no. 15, pp. 1885-1899, 2006.

[63] M. S. Manger, E. Strand, M. Ebbing et al., "Dietary intake of n-3 long-chain polyunsaturated fatty acids and coronary events in Norwegian patients with coronary artery disease," The American Journal of Clinical Nutrition, vol. 92, no. 1, pp. 244-251, 2010.

[64] The ORIGIN Trial Investigators et al., "N-3 fatty acids and cardiovascular outcomes in patients with dysglycemia," The New England Journal of Medicine, vol. 367, pp. 1760-1761, 2012.

[65] F. B. Hu, L. Bronner, W. C. Willett et al., "Fish and omega3 fatty acid intake and risk of coronary heart disease in women," Journal of the American Medical Association, vol. 287, no. 14, pp. 1815-1821, 2002.

[66] K. Oh, F. B. Hu, J. E. Manson, M. J. Stampfer, and W. C. Willett, "Dietary fat intake and risk of coronary heart disease in women: 20 Years of follow-up of the nurses' health study," American Journal of Epidemiology, vol. 161, no. 7, pp. 672679, 2005.

[67] R. B. Singh, M. A. Niaz, J. P. Sharma, R. Kumar, V. Rastogi, and M. Moshiri, "Randomized, double-blind, placebocontrolled trial of fish oil and mustard oil in patients with suspected acute myocardial infarction: the Indian experiment of infarct survival-4," Cardiovascular Drugs and Therapy, vol. 11, no. 3, pp. 485-491, 1997.

[68] D. Kromhout, E. B. Bosschieter, and C. de Lezenne Coulander, "The inverse relation between fish consumption and 20 -year mortality from coronary heart disease," The New England Journal of Medicine, vol. 312, no. 19, pp. 1205-1209, 1985.

[69] M. L. Daviglus, J. Stamler, A. J. Orencia et al., "Fish consumption and the 30-year risk of fatal myocardial infarction," The New England Journal of Medicine, vol. 336, no. 15, pp. 10461053, 1997.

[70] M. T. Streppel, M. C. Ocké, H. C. Boshuizen, F. J. Kok, and D. Kromhout, "Long-term fish consumption and n-3 fatty acid intake in relation to (sudden) coronary heart disease death: the Zutphen study," European Heart Journal, vol. 29, no. 16, pp. 2024-2030, 2008.

[71] M. C. Morris, J. E. Manson, B. Rosner, J. E. Buring, W. C. Willett, and C. H. Hennekens, "Fish consumption and cardiovascular disease in the physicians' health study: a prospective study," American Journal of Epidemiology, vol. 142, no. 2, pp. 166-175, 1995.

[72] A. Ascherio, E. B. Rimm, M. J. Stampfer, E. L. Giovannucci, and W. C. Willett, "Dietary intake of marine n-3 fatty acids, fish intake, and the risk of coronary disease among men," The New England Journal of Medicine, vol. 332, no. 15, pp. $977-$ 982, 1995.

[73] D. Kromhout, B. P. M. Bloemberg, E. J. M. Feskens, M. G. L. Hertog, A. Menotti, and H. Blackburn, "Alcohol, fish, fibre and antioxidant vitamins intake do not explain population differences in coronary heart disease mortality," International Journal of Epidemiology, vol. 25, no. 4, pp. 753-759, 1996.

[74] D. W. T. Nilsen, G. Albrektsen, K. Landmark, S. Moen, T. Aarsland, and L. Woie, "Effects of a high-dose concentrate of n-3 fatty acids or corn oil introduced early after an acute myocardial infarction on serum triacylglycerol and HDL cholesterol," The American Journal of Clinical Nutrition, vol. 74, no. 1, pp. 50-56, 2001.

[75] M. L. Burr, P. A. L. Ashfield-Watt, F. D. J. Dunstan et al., "Lack of benefit of dietary advice to men with angina: results of a controlled trial," European Journal of Clinical Nutrition, vol. 57, no. 2, pp. 193-200, 2003.

[76] P. Pietinen, A. Ascherio, P. Korhonen et al., "Intake of fatty acids and risk of coronary heart disease in a cohort of Finnish men. The Alpha-Tocopherol, Beta-Carotene Cancer Prevention Study," American Journal of Epidemiology, vol. 145, no. 10, pp. 876-887, 1997.

[77] W. S. Harris, "Omega-3 fatty acids: the "Japanese" factor?" Journal of the American College of Cardiology, vol. 52, no. 6, pp. 425-427, 2008.

[78] I. A. Brouwer, P. L. Zock, A. J. Camm et al., "Effect of fish oil on ventricular tachyarrhythmia and death in patients with implantable cardioverter defibrillators: The Study on Omega-3 Fatty Acids and Ventricular Arrhythmia (SOFA) Randomized Trial," Journal of the American Medical Association, vol. 295, no. 22, pp. 2613-2619, 2006.

[79] A. A. Finzi, R. Latini, S. Barlera et al., "Effects of $n-3$ polyunsaturated fatty acids on malignant ventricular arrhythmias in patients with chronic heart failure and implantable cardioverter-defibrillators: a substudy of the Gruppo Italiano per lo Studio della Sopravvivenza nell'Insufficienza Cardiaca (GISSI-HF) trial," American Heart Journal, vol. 161, no. 2, pp. 338-343, 2011.

[80] M. H. Raitt, W. E. Connor, C. Morris et al., "Fish oil supplementation and risk of ventricular tachycardia and ventricular fibrillation in patients with implantable defibrillators: a randomized controlled trial," Journal of the American Medical Association, vol. 293, no. 23, pp. 2884-2891, 2005.

[81] T. Madsen, J. H. Christensen, A. M. Thøgersen, E. B. Schmidt, and E. Toft, "Intravenous infusion of n-3 polyunsaturated fatty acids and inducibility of ventricular tachycardia in patients with implantable cardioverter defibrillator," Europace, vol. 12, no. 7, pp. 941-946, 2010.

[82] H. Aarsetoey, R. Aarsetoey, T. Lindner, H. Staines, W. S. Harris, and D. W. T. Nilsen, "Low levels of the omega-3 index are associated with sudden cardiac arrest and remain stable in survivors in the subacute phase," Lipids, vol. 46, no. 2, pp. 151-161, 2011.

[83] R. Marchioli, F. Barzi, E. Bomba et al., "Early protection against sudden death by $n-3$ polyunsaturated fatty acids after myocardial infarction: time-course analysis of the results of the Gruppo Italiano per lo Studio della Sopravvivenza nell'Infarto Miocardico (GISSI)-Prevenzione," Circulation, vol. 105, no. 16, pp. 1897-1903, 2002.

[84] D. S. Siscovick, T. E. Raghunathan, I. King et al., "Dietary intake and cell membrane levels of long-chain n-3 polyunsaturated fatty acids and the risk of primary cardiac arrest," Journal of the American Medical Association, vol. 274, no. 17, pp. 1363-1367, 1995.

[85] C. M. Albert, H. Campos, M. J. Stampfer et al., "Blood levels of long-chain n-3 fatty acids and the risk of sudden death," The New England Journal of Medicine, vol. 346, no. 15, pp. 1113-1118, 2002.

[86] R. N. Lemaitre, I. B. King, D. Mozaffarian, L. H. Kuller, R. P. Tracy, and D. S. Siscovick, "N-3 polyunsaturated fatty acids, fatal ischemic heart disease, and nonfatal myocardial infarction in older adults: The Cardiovascular Health Study," The American Journal of Clinical Nutrition, vol. 77, no. 2, pp. 319-325, 2003.

[87] S. Pepe and P. L. McLennan, "Dietary fish oil confers direct antiarrhythmic properties on the myocardium of rats," The Journal of Nutrition, vol. 126, no. 1, pp. 34-42, 1996. 
[88] G. E. Billman, J. X. Kang, and A. Leaf, "Prevention of sudden cardiac death by dietary pure $\omega-3$ polyunsaturated fatty acids in dogs," Circulation, vol. 99, no. 18, pp. 2452-2457, 1999.

[89] P. L. McLennan, T. M. Bridle, M. Y. Abeywardena, and J. S. Charnock, "Comparative efficacy of n-3 and n-6 polyunsaturated fatty acids in modulating ventricular fibrillation threshold in marmoset monkeys," The American Journal of Clinical Nutrition, vol. 58, no. 5, pp. 666-669, 1993.

[90] N. R. Matthan, H. Jordan, M. Chung, A. H. Lichtenstein, D. A. Lathrop, and J. Lau, "A systematic review and metaanalysis of the impact of $\omega-3$ fatty acids on selected arrhythmia outcomes in animal models," Metabolism, vol. 54, no. 12, pp. 1557-1565, 2005.

[91] H. Aarsetøy, V. Pönitz, O. B. Nilsen, H. Grundt, W. S. Harris, and D. W. T. Nilsen, "Low levels of cellular omega-3 increase the risk of ventricular fibrillation during the acute ischaemic phase of a myocardial infarction," Resuscitation, vol. 78, no. 3, pp. 258-264, 2008.

[92] L. Hooper, R. L. Thompson, R. A. Harrison et al., "Risks and benefits of omega 3 fats for mortality, cardiovascular disease, and cancer: systematic review," British Medical Journal, vol. 332, no. 7544, pp. 752-760, 2006.

[93] K. B. Filion, F. El Khoury, M. Bielinski, I. Schiller, N. Dendukuri, and J. M. Brophy, "Omega-3 fatty acids in highrisk cardiovascular patients: a meta-analysis of randomized controlled trials," BMC Cardiovascular Disorders, vol. 10, article 24, 2010

[94] S. P. Whelton, J. He, P. K. Whelton, and P. Muntner, "Metaanalysis of observational studies on fish intake and coronary heart disease," American Journal of Cardiology, vol. 93, no. 9, pp. 1119-1123, 2004.

[95] K. He, Y. Song, M. L. Daviglus et al., "Accumulated evidence on fish consumption and coronary heart disease mortality: a meta-analysis of cohort studies," Circulation, vol. 109, no. 22, pp. 2705-2711, 2004.

[96] D. Yzebe and M. Lievre, "Fish oils in the care of coronary heart disease patients: a meta-analysis of randomized controlled trials," Fundamental and Clinical Pharmacology, vol. 18, no. 5, pp. 581-592, 2004.

[97] P. E. Marik and J. Varon, "Omega-3 dietary supplements and the risk of cardiovascular events: a systematic review," Clinical Cardiology, vol. 32, no. 7, pp. 365-372, 2009.

[98] Q. Chen, L. Q. Cheng, T. H. Xiao et al., "Effects of omega3 fatty acid for sudden cardiac death prevention in patients with cardiovascular disease: a contemporary meta-analysis of randomized, controlled trials," Cardiovascular Drugs and Therapy, vol. 25, no. 3, pp. 259-265, 2011.

[99] H. C. Bucher, P. Hengstler, C. Schindler, and G. Meier, "N-3 polyunsaturated fatty acids in coronary heart disease: a metaanalysis of randomized controlled trials," American Journal of Medicine, vol. 112, no. 4, pp. 298-304, 2002.

[100] S. M. Kwak, S. K. Myung, J. Y. Lee, and H. G. Seo, "Efficacy of omega-3 fatty acid supplements (eicosapentaenoic and docohexaenoic acid) in the secondary prevention of cardiovascular disease: a meta-analysis of randomized, double-blind, placebo-controlled trials," Archives of Internal Medicine, vol. 172, no. 9, pp. 686-694, 2012.

[101] D. J. A. Jenkins, A. R. Josse, J. Beyene et al., "Fish-oil supplementation in patients with implantable cardioverter defibrillators: a meta-analysis," Canadian Medical Association Journal, vol. 178, no. 2, pp. 157-164, 2008.

[102] E. C. Rizos, E. E. Ntzani, E. Bika, M. S. Kostapanos, and M. S. Elisaf, "Association between omega-3 fatty acid supplementation and risk of major cardiovascular disease events. A systematic review and meta-analysis," Journal of the American Medical Association, vol. 308, no. 10, pp. 10241033, 2012.

[103] J. Delgado-Lista, P. Perez-Martinez, J. Lopez-Miranda, and F. Perez-Jimenez, "Long chain omega-3 fatty acids and cardiovascular disease: a systematic review," British Journal of Nutrition, vol. 107, supplement 2, pp. S201-S213, 2012.

[104] P. M. Kris-Etherton, W. S. Harris, and L. J. Appel, "Fish consumption, fish oil, omega-3 fatty acids, and cardiovascular disease," Circulation, vol. 106, no. 21, pp. 2747-2757, 2002.

[105] R. Sadovsky and P. Kris-Etherton, "Prescription omega-3acid ethyl esters for the treatment of very high triglycerides," Postgraduate Medicine, vol. 121, no. 4, pp. 145-153, 2009.

[106] A. C. Skulas-Ray, S. G. West, M. H. Davidson, and P. M. Kris-Etherton, "Omega-3 fatty acid concentrates in the treatment of moderate hypertriglyceridemia," Expert Opinion on Pharmacotherapy, vol. 9, no. 7, pp. 1237-1248, 2008.

[107] W. S. Harris, "Omega-3 fatty acids and cardiovascular disease: a case for omega-3 index as a new risk factor," Pharmacological Research, vol. 55, no. 3, pp. 217-223, 2007.

[108] W. S. Harris, "The omega-3 index: from biomarker to risk marker to risk factor," Current Atherosclerosis Reports, vol. 11, no. 6, pp. 411-417, 2009.

[109] R. C. Block, W. S. Harris, K. J. Reid, S. A. Sands, and J. A. Spertus, "EPA and DHA in blood cell membranes from acute coronary syndrome patients and controls," Atherosclerosis, vol. 197, no. 2, pp. 821-828, 2008.

[110] Y. J. Kim, D. W. Jeong, J. G. Lee et al., "Omega-3 index and smoking in patients with acute ST-elevation myocardial infarction taking statins: a case-control study in Korea," Lipids in Health and Disease, vol. 11, article 43, 2012.

[111] H. Aarsetoey, V. Pönitz, H. Grundt, H. Staines, W. S. Harris, and D. W. T. Nilsen, "(N-3) fatty acid content of red blood cells does not predict risk of future cardiovascular events following an acute coronary syndrome," The Journal of Nutrition, vol. 139, no. 3, pp. 507-513, 2009.

[112] P. D. Watson, P. S. Joy, C. Nkonde, S. E. Hessen, and D. G. Karalis, "Comparison of bleeding complications with omega3 fatty acids + aspirin + clopidogrel-versus-aspirin + clopidogrel in patients with cardiovascular disease," American Journal of Cardiology, vol. 104, no. 8, pp. 1052-1054, 2009. 


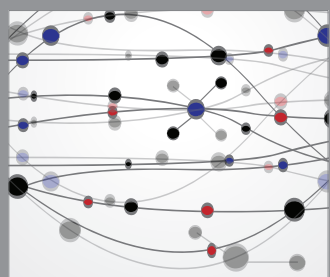

The Scientific World Journal
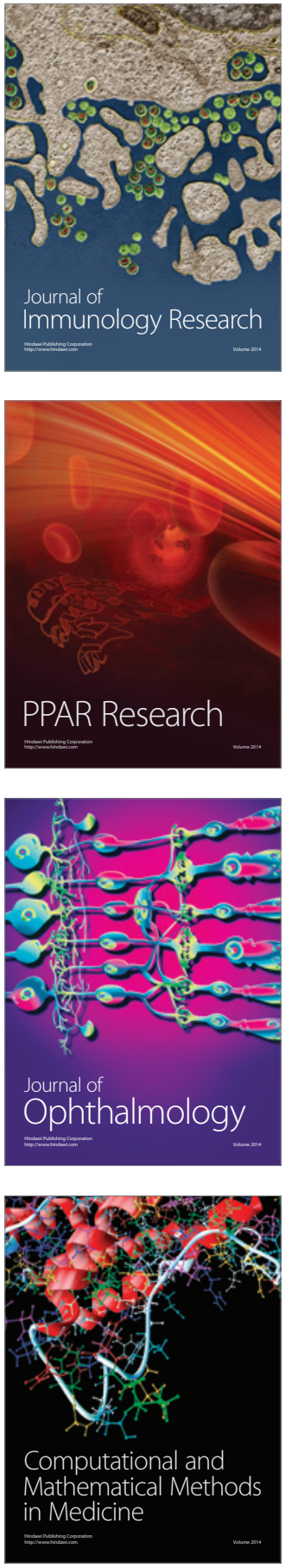

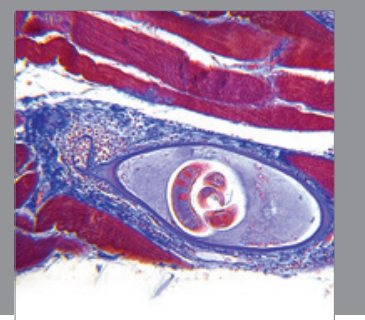

Gastroenterology

Research and Practice
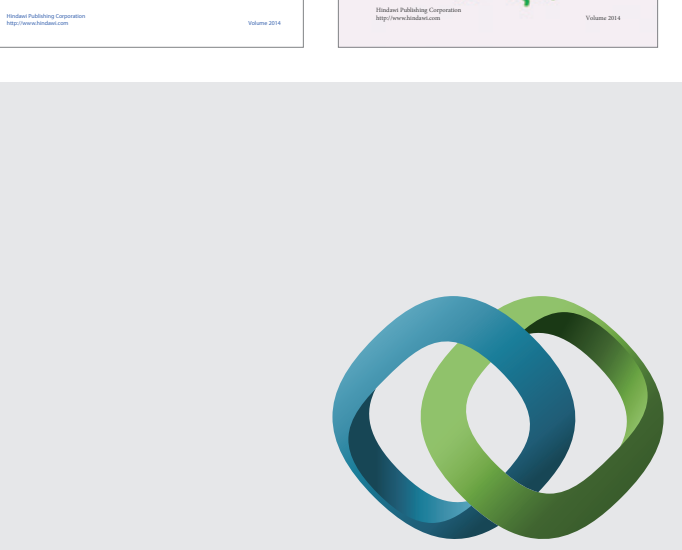

\section{Hindawi}

Submit your manuscripts at

http://www.hindawi.com
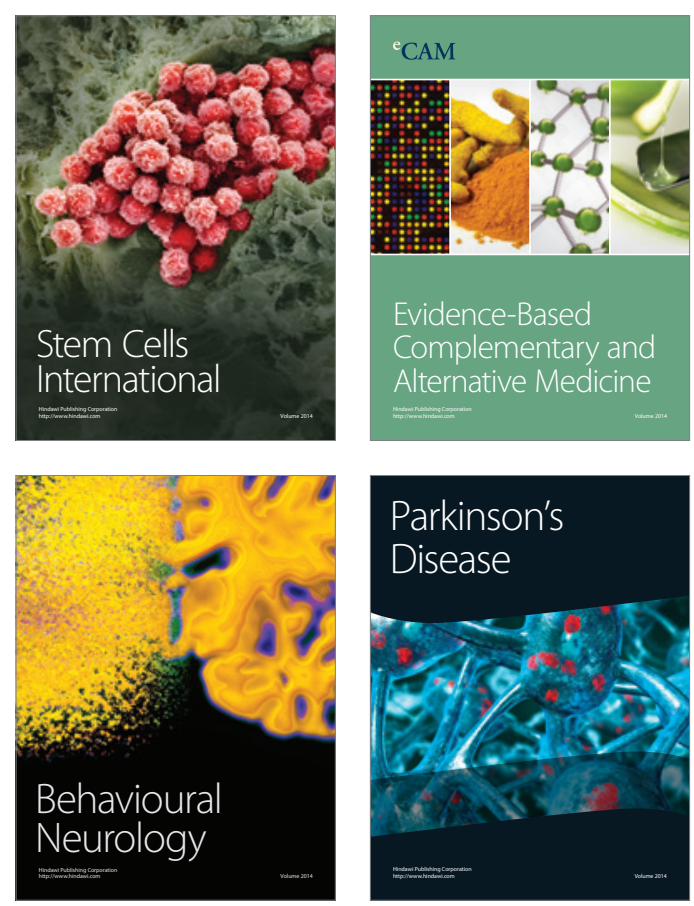

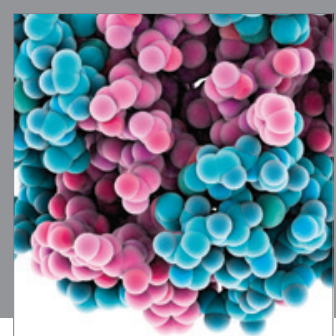

Journal of
Diabetes Research

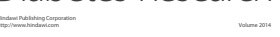

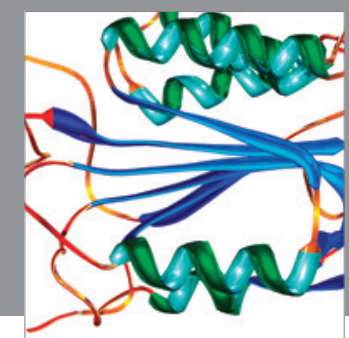

Disease Markers
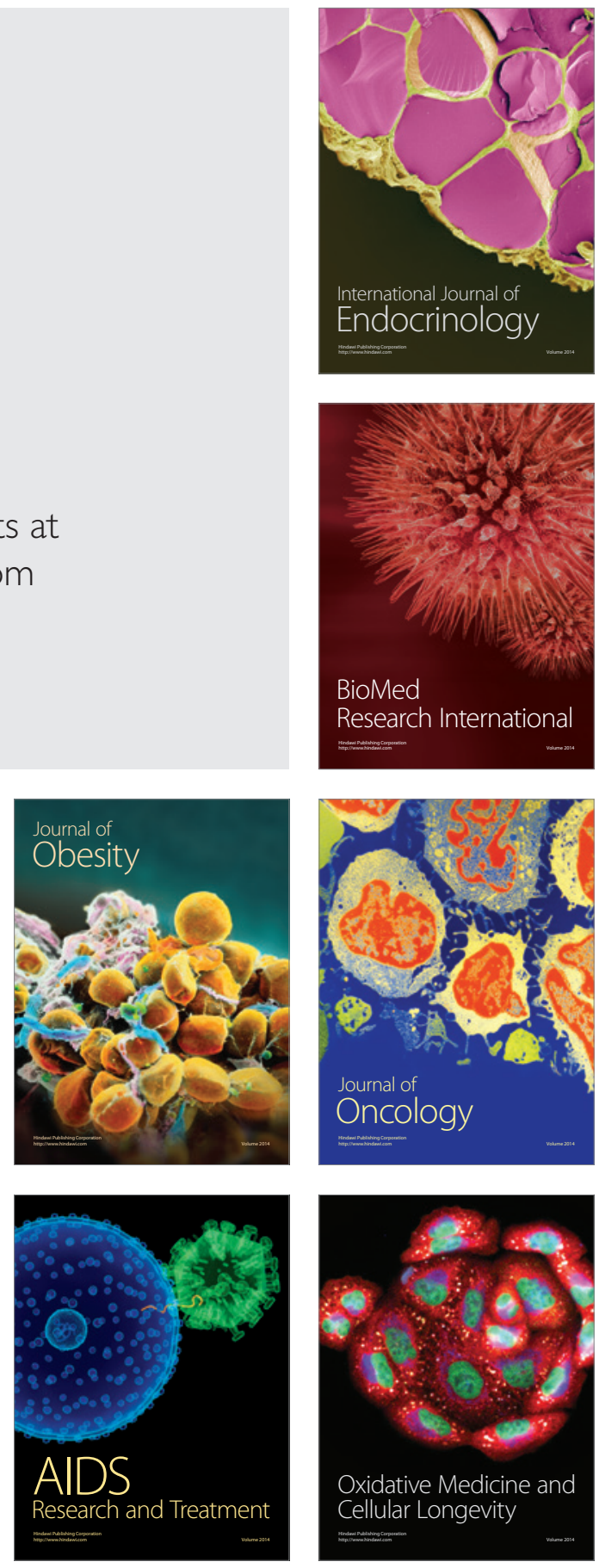